\title{
Effect of combinatorial bone morphogenetic protein 2 and bone morphogenetic protein 7 gene delivery on osteoblastic differentiation
}

\author{
Young Bae ${ }^{1}$, Kyoung-Hwa Kim ${ }^{1}$, Su-Hwan Kim ${ }^{1,2}$, Chul-Woo Lee ${ }^{1}$, Ki-Tae Koo ${ }^{1}$, Tae-Il Kim ${ }^{1}$, \\ Yang-Jo Seol ${ }^{1}$, Young $\mathrm{Ku}^{1}$, In-Chul Rhyu ${ }^{1}$, Chong-Pyoung Chung ${ }^{1}$, Yong-Moo Lee ${ }^{1^{*}}$ \\ 1. Department of Periodontology, School of Dentistry, Seoul National University \\ 2. Department of Periodontics, Asan Medical Center
}

\begin{abstract}
Purpose: Gene therapy (ex vivo) has recently been used as a means of delivering bone morphogenetic proteins (BMPs) to sites of tissue regeneration. In the present study, we investigated the effect of co-transduction of adenoviruses expressing BMP-2 and BMP-7 on osteogenesisof $\mathrm{C} 2 \mathrm{C} 12$ cells in vitro.

Methods: A replication-defective human adenovirus 5 (Ad5) containing a cDNA for BMPs in the E1 region of the virus (Ad5BMP-2 and Ad5BMP-7) was constructed by in vivo homologous recombination. Functional activity of Ad5BMP-2 and Ad5BMP-7 were evaluated in mouse stromal cells (W20-17cells). C2C12 cells are transduced with various MOI (multiplicity of infection) of Ad5BMP-2 and Ad5BMP-7 to assess most effective and stable titer. Based on this result, C2C12 cells were transduced with Ad5BMP-2 and Ad5BMP-7 alone or by combination. BMPs expression, alkaline phosphatase (ALPase) activity, cell proliferation, and mineralization were assessed.

Results: Ad5BMP-2 and Ad5BMP-7 are successfully transduced to W20-17 cells, and secreted BMPs stimulated cell differentiation. Also, $\mathrm{C} 2 \mathrm{C} 12$ cells transduced with Ad5BMPs showed expression of BMPs and increased ALPaseactivity. In all groups, cell proliferation was observed over times. At 7days, cells co-transduced with Ad5BMP-2 and Ad5BMP-7 showed lower proliferation than the others. C2C12 cells co-transduced with Ad5BMP-2 and Ad5BMP-7 had greater ALPaseactivity than that would be predicted if effect of individual Ad5BMPs were additive. Little mineralized nodule formation was detected in cells transduced with individual Ad5BMPs. In contrast, Ad5BMP-2 and Ad5BMP-7 combination stimulated mineralization after culturing for 10 days in mineralizing medium.

Conclusions: Present study demonstrated that adenoviruses expressing BMPs gene successfully produced BMPs protein and these BMPs stimulated cells to be differentiated into osteoblastic cells. In addition, the osteogenic activity of Ad5BMPs can be synergistically increased by co-transduction of cells with Ad5BMP-2 and Ad5BMP-7.
\end{abstract}

(J Korean Acad Periodontol 2009;39:279-286)

KEY WORDS: bone morphogenetic protein 2; bone morphogenetic protein 7; gene therapy; osteogenesis.

\section{INTRODUCTION}

The growth factors that are known to affect osteogenic cells and may have therapeutic potential in promoting periodontal regeneration include platelet-derived growth factors, fibroblast growth factors,

Correspondence: Dr. Yong-Moo Lee

Department of Periodontology, School of Dentistry, Seoul National University, 28, Yongon-dong, Chongno-Ku, Seoul, 110-744, Korea. E-mail: ymlee@snu.ac.kr, Tel: 82-2-2072-3024, Fax: 82-2-744-0051. Received: Jul 21, 2009; Accepted: Jul 28, 2009

This work was supported by the Korea Science and Engineering Foundation (KOSEF) grant funded by the Korea government (MOST) (No. M10646010003-08N4601-00310). and transforming growth factors. They have been suggested as suitable agents because of their ability to regulate cell proliferation in differentiated tissue $^{1-4)}$. However, growth factors are not capable of inducing bone formation in ectopic sites. Rather, they act to modulate or stimulate already determined osteoprogenitor cells in developing or pre-existing orthotopic sites to form cartilage and bone ${ }^{1,3)}$.

Unlike these growth factors, Bone morphogenetic proteins (BMPs) have osteoinductive activity. When BMPs bind to their cell surface receptors on mesenchymal cell, a BMP signaling cascade is activated. 
Signals are sent via specific genes that lead to the synthesis of macromolecules involved in cartilage and bone formation, and the mesenchymal cell becomes a chondrocyte or an osteoblast ${ }^{5)}$. There is, therefore, a distinct difference between the ability and function of BMPs and growth factors.

However, human trials with BMPs have generally been less successful than earlier animal studies ${ }^{6}$. Reasons for this may be related to a lack of optimal matrix for controlled, sustained BMP delivery at the site of implantation, short biologic half life, and inability of recombinant molecule presentation after implantation to mimic the route of administration in vivo by a BMP-producing cell ${ }^{7-8)}$.

Gene therapy has recently been used as a means of delivering BMPs to sites of tissue regeneration. The strategy is to accelerate or induce a natural biologic process by expressing a molecule that is normally involved in the regenerative response for the tissue of interest. There are two general ways to deliver a gene to a regenerative site: BMP gene can be delivered directly to the tissue of interest where it will be taken up by host cells that will then express the regenerative molecule (in vivo) or the gene is transduced into the cells of a tissue biopsy outside the body, and then resulting genetically modified cells are transplanted back into patient (ex vivo) ${ }^{8-10)}$.

Although individual BMPs can induce bone formation, there is strong evidence that these factors normally work together to induced bone formation. BMPs 2, 4 and 7 are expressed in overlapping patterns during limb development ${ }^{11)}$ and observed at various times during fracture healing ${ }^{12)}$. Previous studies suggest that BMP gene therapy may be more effective if it is used to express combinations of BMPs rather than single molecules ${ }^{13-14)}$.

In the present study, we evaluated adenovirus vector for BMPs gene delivery and investigated the effect of co-transduction of adenoviruses expressing BMP-2 and $\mathrm{BMP}-7$ on osteoblastic differentiation of $\mathrm{C} 2 \mathrm{C} 12$ cells in vitro.

\section{MATERIALS AND METHODS}

\section{Adenovirus vector construction}

A replication-defective human adenovirus 5 (Ad5) containing a cDNA for BMPs in the E1 region of the virus (Ad5BMP-2 and Ad5BMP-7, respectively) was constructed by in vivo homologous recombination. Briefly, expression cassette containing BMP gene (BMP-2 or BMP-7) was amplified in E. coli, and ligated to Adeno-X Viral DNA (BD Biosciences Clontech, San Jose, USA). Recombinant adenoviruses derived from Adeno-X Viral DNA were propagated in human embryonic kidney (HEK) 293 cells and titrated. A replication-defective human adenovirus 5 containing a cDNA for LacZ (AdLacZ) was used as a negative control vector because this vector does not stimulate bone formation.

\section{Cell culture}

W20-17 (mouse stromal cell line) cells and C2C12 (murine myoblastic cell line) cells were used in this study. W20-17 cells were used for evaluation of functional activity of adenoviral vector, Ad5BMPs. C2C12 cells were used for examining co-operative interactions between Ad5BMP-2 and Ad5BMP-7. Cells were maintained in Dulbeccos modified eagles medium (DMEM, Gibco, Carlsbad, USA) containing 10\% fetal bovine serum (FBS), and $1 \%$ penicillin-streptomycin solution (5,000 units $/ \mathrm{ml}$ penicillin and $50 \mu \mathrm{g} / \mathrm{ml}$ streptomycin, Gibco, Carlsbad, USA) at $37{ }^{\circ} \mathrm{C}$ in a humidified atmosphere of $95 \%$ air and $5 \% \mathrm{CO}_{2}$.

\section{Adenoviral vector transduction}

W20-17 cells were transduced with Ad5BMP-2 or Ad5BMP-7 to determine whether Ad5BMPs have functional activity. Four days later, enzyme linked immunosorbent assay (ELISA) for BMP-2 or BMP-7 pro- 
tein expression and ALPase activity assessment were performed.

Ad5BMP-2 and Ad5BMP-7 were transduced into C2C12 cells with various MOI (multiplicity of infection) to determine effective and stable titer. Based on this result, C2C12 cells were transduced with Ad5BMP-2 and Ad5BMP-7 alone or by combination. C2C12 cells were plated at a density of 50,000 cells/well for ALPase activity and BMPs expression, and 5,000 cells/well for MTT. Cells were transduced with Ad5BMPs at the indicated titer. After 4hrs, media was added to cells.

\section{Identification and quantitation of BMPs ex- pression}

Conditioned media from transduced cells was collected up to 18 days for quantitation of BMPs. The amount of BMPs present in the media was quantified using commercial enzyme immunoassay kit (R\&D system, Minneapolis, USA).

\section{Cell proliferation assay}

3-(4, 5- dimethylthiazol-2yl)-diphenyltetrazolium bromide (MTT) (sigma, St. Louis, USA) assay performed to assess toxicity of Ad5BMPs and secreted BMPs. MTT solution was added to each sample at 1, 3, 7 days after transduction. After 4 hours, dimethyl sulfoxide (DMSO) added for extraction of formazon. The optical density of each extraction solution was measured with an automatic microplate reader (Molecular Devices, Sunnyvale, USA) at a wavelength of $540 \mathrm{~nm}$.

\section{Measurement of alkaline phosphatase (ALPase) acitivity}

After transduction to $\mathrm{C} 2 \mathrm{C} 12$ cells with Ad5BMP-2 and Ad5BMP-7 alone or by combination, each cells were collected at 3, 7 and 14 days. The cells lysised with lysis buffer and centrifuged. The supernatant was collected and incubated with $0.1 \mathrm{M}$ Glycine, $0.1 \%$ Triton X-100, $15 \mathrm{mM}$ pNPP for 30min and stopped by adding $0.1 \mathrm{~N} \mathrm{NaOH}$. The absorbance of the reaction was measured at $405 \mathrm{~nm}$ in a microplate reader.

\section{Mineralization assay}

The effect of Ad5BMPs on mineralized nodule formation was determined using alizarin red-S (AR-S) staining. C2C12 cells transduced with Ad5BMPs were incubated in mineralizing media (DMEM with $15 \% \mathrm{FBS}$, $1 \%$ penicillin-streptomycin solution, $0.5 \%$ ascorbic acid and $1 \% \beta$-glycerophosphate). After 10 days, Alizarin red-s staining was performed, and then, stained cells were photographed followed by a quantitative eluting procedure using cetylpyridinium chloride in $10 \mathrm{mM}$ sodium phosphate.

\section{Statistical analysis}

Statistical analyses were performed using oneway ANOVA analysis. Statistical significance was set at $P<0.05$.

\section{RESULTS}

\section{Functional activity of Ad5BMPs}

The functional activity of Ad5BMPs was evaluated in mouse W20-17 cells. BMP-2 and BMP-7 protein secretion was assessed by ELISA (Fig. 1). Cells transduced with Ad5BMP-2 secreted BMP-2 protein. Cells transduced with Ad5BMP-7 secreted BMP-7 protein. In contrast, no BMPs protein was detected in uninfected cells.

The ability of Ad5BMP-2 and Ad5BMP-7 to stimulate the early osteoblast marker, ALPase activity was examined (Fig. 2). Compared to cells uninfected, cells transduced with Ad5BMP-2 or Ad5BMP-7 showed enhanced ALPase activity. Ad5BMP-2 was found to more 


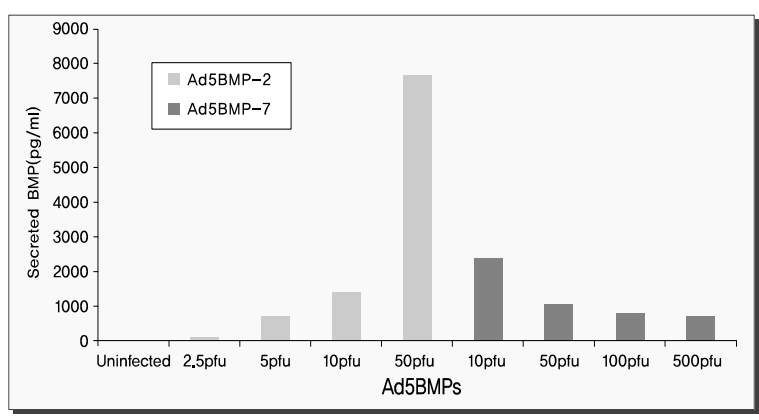

Figure 1. Quantitation of BMP-2 and BMP-7 expression in conditioned media for W20-17 cells at 4 days after Ad5BMPs transduction. * Ad5BMP-2: cells transduced with a replication- defective human adenovirus 5 containing a cDNA for BMP-2, Ad5BMP-7: cells transduced with a replication- defective human adenovirus 5 containing a CDNA for BMP-7.

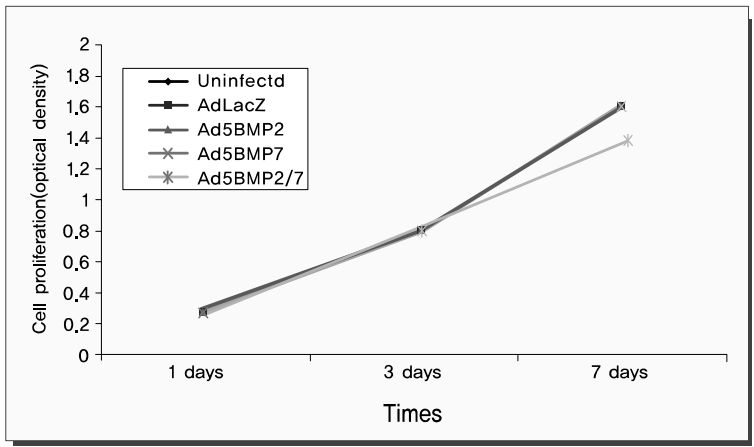

active in stimulating ALPase activity relative to Ad5BMP-7.

\section{Effect of Ad5BMPs on proliferation of the cells}

In both control and test group, $\mathrm{C} 2 \mathrm{C} 12$ cell proliferation was observed over times. In all groups, there were statistically significant differences between culture periods. In addition, there were no statistically significant differences between groups but in group combining Ad5BMP-2 with Ad5BMP-7 at 7days (Fig. 3).

\section{Co-operative interaction of Ad5BMP-2 \& Ad5BMP-7}

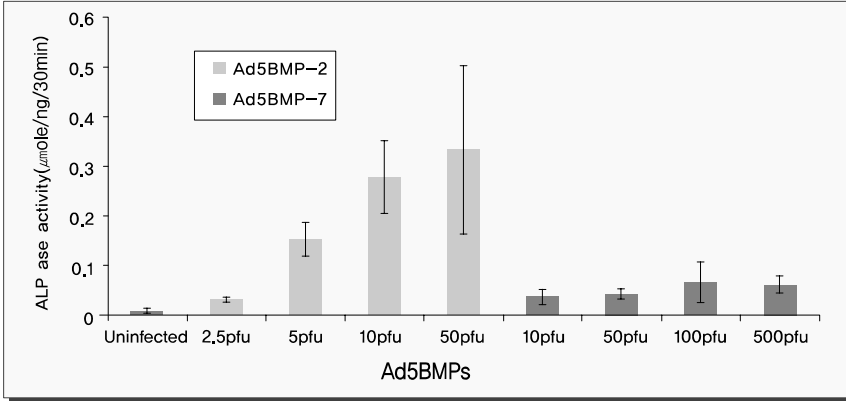

Figure 2. Alkaline phosphatase (ALPase) activity by transduction of W20-17 cell with Ad5BMP-2 or Ad5BMP-7 at 4 days after Ad5BMPs transduction. * Ad5BMP-2: cells transduced with a replication-defective human adenovirus 5 containing a CDNA for BMP-2, Ad5BMP-7: cells transduced with defective human adenovirus 5 containing a CDNA for BMP-7.

Figure 3. Proliferation of C2C12 cells during 7 days. Statistically significant differences were between cells tranduced with Ad5BMP-2/7 combination and the others at 7days (P Y0.05). * Uninfected: untransfected cells, AdLacZ: cells transduced with a replication-defective human adenovirus 5 containing a cDNA for LacZ, Ad5BMP-2: cells transduced with a replication-defective human adenovirus 5 containing a CDNA for BMP-2, Ad5BMP-7: cells transduced with a replication-defective human adenovirus 5 containing a cDNA for BMP-7, Ad5BMP-2/7: cells co-transduced with a replication-defective human adenovirus 5 containing a CDNA for BMP-2 and BMP-7.

Before evaluating whether co-operative interaction can be detected in cultured myoblastic cell, C2C12, we initially determined optimal M.O.I. of Ad5BMPs for transduction. C2C12 cells were transduced with Ad5BMP-2 or Ad5BMP-7 at various M.O.I. Seven days later, BMPs protein secretion and ALPase activity were assessed (Data not shown).

Ad5BMP-2 expressed adequate BMP-2 protein and stimulated ALPase activity at M.O.I. of $7 \mathrm{pfu}$. While, Ad5BMP-7 showed good result at M.O.I. of $3 \mathrm{pfu}$. Based on these results, 7 pfu of Ad5BMP-2 and 3 pfu of Ad5BMP-7were used. 10 pfu of AdLacZ was used for negative control.

C2C12 cells transduced with Ad5BMP-2 alone and co-transduced with Ad5BMP-2 and Ad5BMP-7 secreted BMP-2 protein. Cells uninfected, transduced 


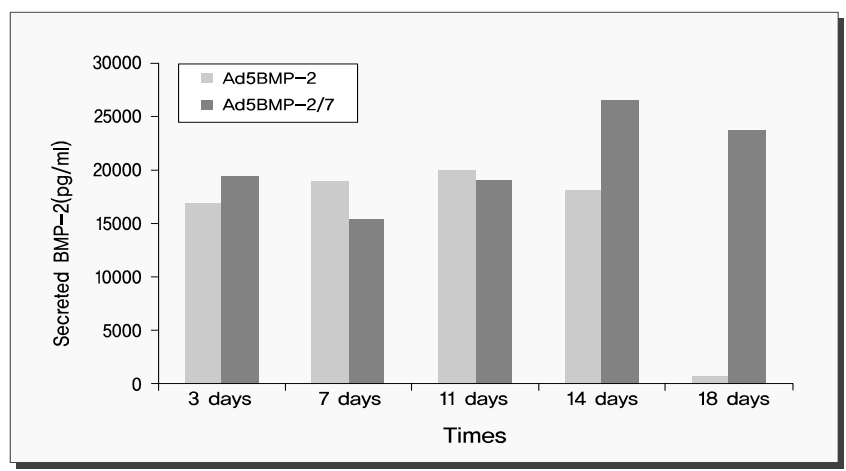

Figure 4. Quantitation of BMP-2 expression in conditioned media during 18 days. The cells transduced with Ad5BMP-2 alone and co-transduced with Ad5BMP-2 and Ad5BMP-7 secreted BMP-2 protein. In conditioned media from cells transduced with ad5BMP-2 alone, similar amount of BMP-2 was observed up to 14days. BMP-2 expression decreased significantly at 18days. Cells co-transduced with Ad5BMP-2 and Ad5BMP-7 secreted BMP-2 protein constantly up to 18 days. *Ad5BMP-2: cells transduced with a replication-defective human adenovirus 5 containing a cDNA for BMP-2, Ad5BMP-2/7: cells co-transduced with a replication-defective human adenovirus h5 containing a CDNA for BMP-2 and BMP-7.

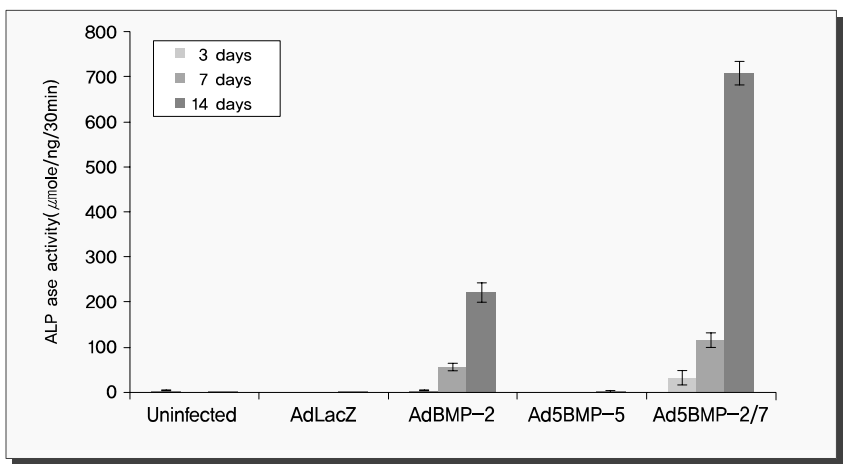

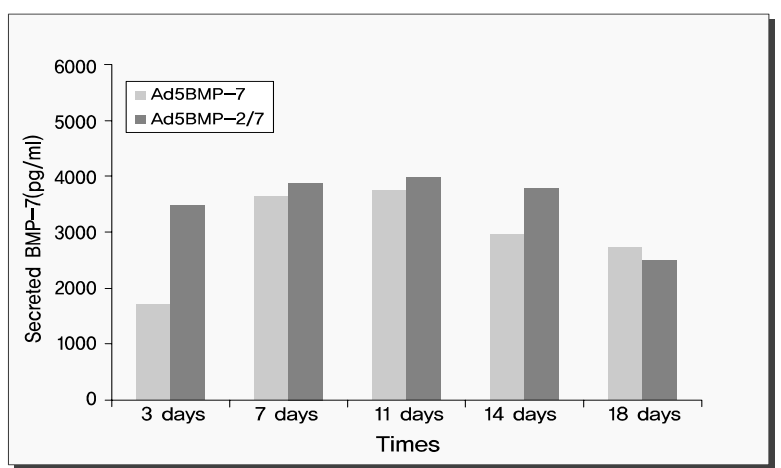

Figure 5. Quantitation of BMP-7 expression in conditioned media for C2C12 cells during 18 days. Cells co-transduced with Ad5BMP-2 and Ad5BMP-7 secreted BMP-7 protein up to 18 days. * Ad5BMP-7: cells transduced with a replication-defective human adenovirus 5 containing a cDNA for BMP-7, Ad5BMP-2/7: cells co-transduced with a replication-defective human adenovirus 5 containing a CDNA for BMP-2 and BMP-7.

replication-defective human adenovirus 5 containing a cDNA for BMP-7. Ad5BMP-2/7: cells co-transduced with a replication-defective human adenovirus 5 containing a CDNA for BMP-2 and BMP-7.

with AdLacZ or Ad5BMP-7 alone showed no expression of BMP-2. Up to 14 days, similar amount of BMP-2 was observed in conditioned media from cells transduced with Ad5BMP-2 alone. However, BMP-2 expression decreased significantly at 18days. Cells cotransduced with Ad5BMP-2 and Ad5BMP-7 secreted BMP-2 protein constantly up to 18 days (Fig. 4).

BMP-7 expression was observed in the conditioned media from cells transduced with Ad5BMP-7 alone or co-transduced with Ad5BMP-2 and Ad5BMP-7. Up to 18 days, BMP-7 level was maintained relatively (Fig. 5).

Cells transduced with Ad5BMP-2 showed increase of ALPase activity over times. However, Ad5BMP-7 did not stimulate ALPase activity compared to control. Nevertheless, combining Ad5BMP-2 with Ad5BMP-7 synergistically stimulated ALPase activity. Cells co-transduced with Ad5BMP-2 and Ad5BMP-7 had greater ALPase activity than that would be predicted if effect of individual Ad5BMPs were additive. ALPase activity in cells treated with Ad5BMP-2 plus 
Ad5BMP-7 was 3 fold greater than the sum of ALPase activity in cells individually treated with Ad5BMP-2 or Ad5BMP-7 at 14 days (Fig. 6).

As shown photographs of alizarin red-S stained culture plates (Fig. 7), little mineralized nodule formation was detected in cells transduced with individual Ad5BMPs. In contrast, Ad5BMP-2 and Ad5BMP-7 combination stimulated mineralization after culturing for 10 days in mineralizing media.
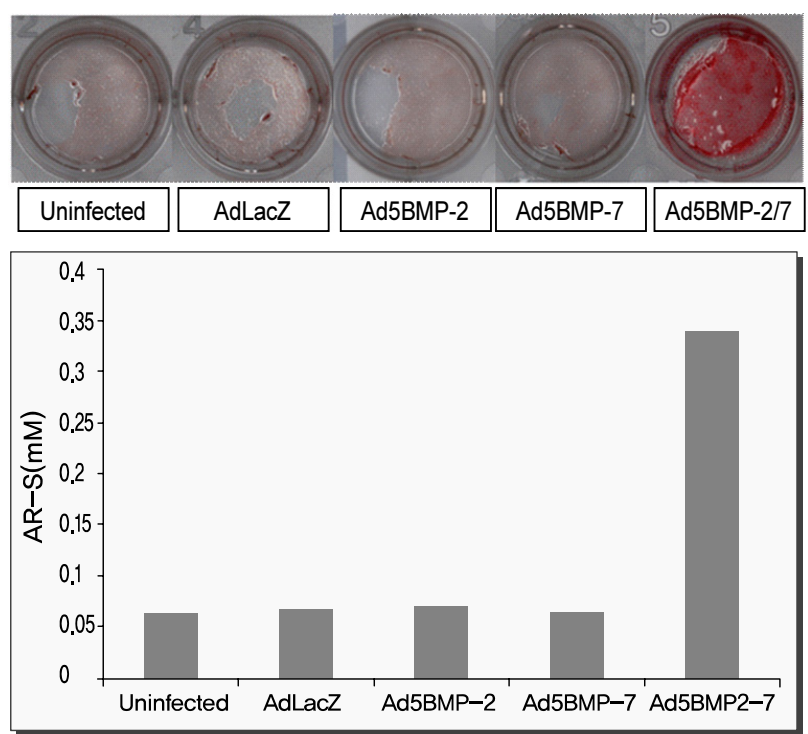

Figure 7. Mineralization assessment. The cells transduced with ad5BMPs were in mineralizing media and stained with alizarin red-S 10days post-transduction. Upper panel showed alizarin-red $S$ stained cells (original magnification $5 X)$. Lower panel presented concentration of alizarin red-S (AR-S) eluted from cells. * Uninfected: untransfected cells, AdLacZ: cells transduced with a replication-defective human adenovirus 5 containing a CDNA for LacZ, Ad5BMP-2. cells transduced with a replication-defective human adenovirus 5 containing a cDNA for BMP-2, Ad5BMP-7: cells transduced with a replication-defective human adenovirus 5 containing a CDNA for BMP-7. Ad5BMP-2/7: cells co-transduced with a replication-defective human adenovirus 5 containing a CDNA for BMP-2 and BMP-7.

\section{DISCUSSION}

BMPs include a large number of proteins belong to the TGF- $\beta$ superfamily and are characterized by their ability to induce bone and cartilage formation ${ }^{1)}$. Urist et al. ${ }^{15)}$ discovered that demineralized, lyophilized rabbit bone induced new bone formation in intramuscular sites. Following the discovery of BMPs in the bone induction model, much knowledge has subsequently been gained with respect to BMPs in the field of embryology ${ }^{5}$. Furthermore, studies have demonstrated the expression of BMPs during tooth development and periodontal repair including alveolar bone ${ }^{16)}$.

King et al. ${ }^{17)}$ stated that rhBMP-2 stimulate cell recruitment, by increasing proliferation and migration of cells from the adjacent unwounded PDL, into the wounded area, thus promoting periodontal regeneration by increasing new cementum formation. According to Fiorellini et al. ${ }^{18)}$, rhBMP-2 delivered by a bioabsorbable collagen sponge revealed significant bone formation in a human buccal wall defect model after tooth extraction when compared with collagen sponge alone.

In general, the impact of a topical delivery of BMPs to periodontal wounds has shown to be promising, yet insufficient for the promotion of predictable periodontal tissue engineering. BMPs proteins, once delivered to the target site, tend to suffer from instability and quick dilution, presumably because of proteolytic breakdown, receptor-mediated endocytosis, and solubility of the delivery vehicle. Because their half lives are significantly reduced, the period of exposure may not be sufficient to act on osteoblasts, cementoblasts, or PDL cells. Different methods of BMPs delivery must be considered ${ }^{8)}$.

Gene transfer methods may circumvent many of the limitations with protein delivery. Gene therapy involves the transfer of genetic information to target cells, which enables them to synthesize a protein of interest to treat disease. Gene transfer is accomplished through the use of viral and non-viral vectors. Examples of viral vectors are retroviruses, adenoviruses and adeno-associated viruses, and non-viral vectors include plasmids and DNA polymer 
complexes. Adenovirus contains DNA, which is introduced into the target cell and subsequently transferred into its nucleus. In contrast to the fate of the retrovirus DNA copy, the adenovirus DNA is not incorporated into the genetic material of host cells. Consequently, when the adenovirus infected target cell divides later, its descendants are not genetically altered, nor do they contain the adenovirus DNA genetic material $^{8-9)}$.

In present study, adenovirus vector containing BMP-2 or BMP-7 gene was evaluated for ex vivo BMPs gene transfer. When non-osteogenic cells, W20-17 or C2C12, were transduced with Ad5BMP-2 or Ad5BMP-7, cells successfully secreted BMP-2 or BMP-7 protein. In addition, secreted BMPs stimulate these non-osteogenic cells to be differentiated into bone-forming cells. In contrast, cells non-transduced or transduced with AdLacZ did not express BMPs protein and showed little ALPase activity.

Many studies about BMPs gene delivery using adenovirus vector have been investigated. Kim et al. $\left.{ }^{19}\right)$ stated that gingiva fibroblast and PDL cell transduced with Ad5BMP-2 were responsive to BMP-2 and showed enhanced ALPase activity and mineralization. According to Franceschi et al. ${ }^{10)}$, cells transduced with AdCMV-BMP7 in vitro produced immunoreactive BMP that was electrophoretically indistinguishable from recombinant protein. Furthermore, this virus could induce the transdifferentiation of $\mathrm{C} 2 \mathrm{C} 12$ into osteoblast like cell in vitro.

When the ALPase activity of Ad5BMPs was examined in $\mathrm{C} 2 \mathrm{C} 12$ cells, Ad5BMP-2 was found to fairly active relative to control. However, Ad5BMP-7 had little or no activity in $\mathrm{C} 2 \mathrm{C} 12$ cells. This is consistent with previous studies. Cheng et al. ${ }^{20)}$ recently reported that adenovirus expressing BMP-2 has stronger ALPaseinducing activity than adenovirus expressing BMP-4 and -7 in C3H10T1/2 and $\mathrm{C} 2 \mathrm{C} 12$ cells. According to Zhao et al. ${ }^{13)}$, in three mesenchymal cell lines (C2H10T1/2, ST2, C2C12), transduction with AdBMPs resulted in only a modest induction of osteoblast markers with AdBMP-2 having higher activity than AdBMP-4 or AdBMP-7.

In present experiment, co-transduction of Ad5BMP-2 and Ad5BMP-7 synergistically stimulated ALPase activity and mineralized nodule formation in $\mathrm{C} 2 \mathrm{C} 12$ cells. Although cells transduced with Ad5BMP-7 only had little effect on ALPase activity, combining of Ad5BMP-2 and Ad5BMP-7 showed enhanced ALPase activity. Synergistic effect of combinatorial BMPs gene delivery was presented by previous studies. In a related study, Zhu et al. ${ }^{14)}$ reported that conditioned media from A549 epithelial cells transduced with AdBMP-2 plus AdBMP-7 was more active in stimulating osteocalcin and ALPase levels in C2C12 cells than conditioned media from cells individually transduced with AdBMP-2 or AdBMP-7 or equivalent amounts of purified recombinant BMP-2 or BMP-7. Zhao et al. ${ }^{13)}$ stated that the osteogenic activity of AdBMPs can be dramatically increased by co-transduction of cells with AdBMP-2/7 and AdBMP-4/7 combinations. Because of their increased biologic activity, AdBMPs combination can achieve bone regeneration at much lower viral titers, thereby minimizing possible toxicity.

BMPs can be divided into subgroup based on similarities in their amino acid sequences. BMP-2 and BMP-7 share only slightly greater than 50\% homology at the amino acid level, and belong to different subgroup and exhibit different receptor binding pro perties. While BMP-2 mainly binds to the type I receptors, actinon receptor-like kidnaps 3 and ILK-6, BMP-7 preferentially binds to ILK $-2^{11}$. These differences between BMP-2 and BMP-7 can be reason for synergistic effect of BMP-2 and BMP-7 combination.

Francesca et al. ${ }^{10)}$ stated that co-transaction of mesenchymal cell lines with adenovirus expressing combination of BMPs produced BMP heterodimers and these have greater biologic activity than homodimer. However, the authors said that because they have not determined the stoichiometry of BMP-2/7 complex, it 
cannot be firmly concluded that these complexes are exclusively heterodimeric.

In conclusion, present results demonstrated that adenoviruses expressing BMPs gene successfully produced BMPs protein and these BMPs stimulated cells to be differentiated into osteoblastic cells. Also the osteogenic activity of Ad5BMPs can be synergistically increased by co-transduction of cells with Ad5BMP-2 and Ad5BMP-7.

\section{REFERENCES}

1. Lee MB. Bone morphogenetic proteins: background and implications for oral reconstruction. A review. Journal of clinical periodontology 1997;24:355-365.

2. Graves DT, Cochran DL. Periodontal regeneration with polypeptide growth factors. Curr Opin Periodontol 1994:178-186.

3. Wozney JM. The potential role of bone morphogenetic proteins in periodontal reconstruction. J Periodontol 1995;66: 506-510.

4. Khan SN, Bostrom MP, Lane JM. Bone growth factors. Orthop Clin North Am 2000;31:375-388.

5. Reddi AH. Bone morphogenetic proteins: from basic science to clinical applications. J Bone Joint Surg Am 2001;83-A Suppl 1:S1-6.

6. Winn SR, Hu Y, Sfeir C, Hollinger JO. Gene therapy approaches for modulating bone regeneration. Adv Drug Deliv Rev 2000;42:121-138.

7. Anusaksathien O, Giannobile WV. Growth factor delivery to re-engineer periodontal tissues. Curr Pharm Biotechnol 2002;3:129-139.

8. Ramseier CA, Abramson ZR, Jin Q, Giannobile WV. Gene therapeutics for periodontal regenerative medicine. Dent Clin North Am 2006;50:245-263.

9. Karthikeyan BV, Pradeep AR. Gene therapy in periodontics: a review and future implications. J Contemp Dent Pract 2006;7:83-91.

10. Franceschi RT, Yang S, Rutherford RB, Krebsbach PH,
Zhao M, Wang D. Gene therapy approaches for bone regeneration. Cells Tissues Organs 2004;176:95-108.

11. Nishimatsu S, Thomsen GH. Ventral mesoderm induction and patterning by bone morphogenetic protein heterodimers in Xenopus embryos. Mech Dev 1998;74:75-88.

12. Cho TJ, Gerstenfeld LC, Einhorn TA. Differential temporal expression of members of the transforming growth factor beta superfamily during murine fracture healing. J Bone Miner Res 2002;17:513-520.

13. Zhao M, Zhao Z, Koh JT, Jin T, Franceschi RT. Combinatorial gene therapy for bone regeneration: cooperative interactions between adenovirus vectors expressing bone morphogenetic proteins 2, 4, and 7. J Cell Biochem 2005;95:1-16.

14. Zhu W, Rawlins BA, Boachie-Adjei O, et al. Combined bone morphogenetic protein-2 and -7 gene transfer enhances osteoblastic differentiation and spine fusion in a rodent model. J Bone Miner Res 2004;19:2021-2032.

15. Urist MR. Bone: formation by autoinduction. Science 1965; 150:893-899.

16. Amar S, Chung KM, NamSH, Karatzas S, Myokai F, Van Dyke TE. Markers of bone and cementum formation accumulate in tissues regenerated in periodontal defects treated with expanded polytetrafluoroethylene membranes. J Periodontal Res 1997;32:148-158.

17. King GN, Hughes FJ. Bone morphogenetic protein-2 stimulates cell recruitment and cementogenesis during early wound healing. Journal of clinical periodontology 2001;28: $465-475$

18. Fiorellini JP, Howell TH, Cochran D, et al. Randomized study evaluating recombinant human bone morphogenetic protein-2 for extraction socket augmentation. J Periodontol 2005;76:605-613.

19. Kim KH, Park YJ, Lee SC et al. A study of bone morphogenetic protein(BMP)-2 gene transduced periodontal cells for bone formation in vitro. J Kor Academy Periodontol 2005;35:511-524.

20. Cheng H, Jiang W, Phillips FM, et al. Osteogenic activity of the fourteen types of human bone morphogenetic proteins (BMPs). J Bone Joint Surg Am 2003;85-A:1544-1552. 